\title{
Designing a high speed 1310nm AlGalnAs/AIGalnAs VCSEL using MgO/Si top DBR and GalnAsP/InP bottom DBR
}

\author{
Arnob Islam ${ }^{1}$, Saiful Islam ${ }^{2}$ \\ ${ }^{1}$ Department of Electrical and Electronic Engineering, Northern University Bangladesh, Dhaka 1213, Bangladesh \\ ${ }^{2}$ Department of Electrical and Electronic Engineering, Bangladesh University of Engineering and Technology, Dhaka 1205, Bangladesh \\ Email address: \\ arnobislam@ymail.com (A. Islam), sislamk@yahoo.com (S. Islam)
}

To cite this article:

Arnob Islam, Saiful Islam. Designing a High Speed 1310nm AlGaInAs/AlGaInAs VCSEL using MgO/Si Top DBR and GaInAsP/InP Bottom DBR. American Journal of Optics and Photonics. Vol. 2, No. 3, 2014, pp. 37-44. doi: 10.11648/j.ajop.20140203.14

\begin{abstract}
In this paper, a 1310nm intracavity structure Vertical Cavity Surface Emitting Laser (VCSEL) has been designed using quaternary compound material of AlGaInAs in both QW and barrier but with different composition. This choice has been made instead of choosing widely used GaInAsP/ InP, GaInAsN/ GaAs to gain some advantages. This combination has shown good band offset in the conduction band. Lattice matching has been obtained in the layers from the substrate up to the top contact layer except the quantum well (QW) layers where small amount of compressive strain of $1.55 \%$ has been used. From the substrate up to the top contact layer, fabrication can be done by epitaxial growth without any difficulty. Reduction in height by using 5 pairs of the top dielectric DBR mirror system of $\mathrm{MgO} / \mathrm{a}-\mathrm{Si}$ is an attraction of this design which can be fabricated by evaporation technique. Dissipation in the bottom DBR due to current flow has been eliminated by using intracavity structure which also gave a way out for the current flow bypassing the dielectric top DBR. The active material compositions have been chosen to obtain a peak gain at $1310 \mathrm{~nm}$. The end result of this design is a top emitting VCSEL based on InP substrate using a different structure which is capable of producing 1310nm light output and which can be constructed easily using widely used epitaxial techniques mixed with the evaporation technique for the top DBR mirror system. The structure is suitable for use in optical ICs.
\end{abstract}

Keywords: Diode Laser, VCSEL, MQW, DBR

\section{Introduction}

Low loss in optical fibers, low dispersion for $1.3 \mu \mathrm{m}$ optical fibers, higher eye safe maximum limit power and lower operation voltage are some of the advantages of long wavelength VCSELs over $850 \mathrm{~nm}$ and 980nm GaAs-based VCSELs [1].

Semiconductor diode lasers producing light output in the $1300 \mathrm{~nm}$ and $1550 \mathrm{~nm}$ windows have great demand in optical fiber communication systems compared to the $980 \mathrm{~nm}$ diode lasers because of their propagation capability over longer distance. These long wavelength lasers are much attractive for low-cost and high-capacity optical interconnects and local access networks. Due to well known advantages of VCSELs current trend has been to replace the edge emitting lasers by VCSELs [2].

GaInAsP/ InP quantum well (QW) based semiconductor active layers have been a common material combination for making VCSELs for the $1300 \mathrm{~nm}$ and $1550 \mathrm{~nm}$ windows because of the matching bandgap of the material. However, GaInAsP QWs, the commonly used gain medium for long-wavelength lasers have lower gain and poorer temperature performance than GaAs and InGaAs QWs on GaAs [1]. The conduction band offset obtained using this combination is low which is not desirable. GaInAsP/ GaInAsP is just a little modification which produces almost similar result as mentioned above.

For the $1300 \mathrm{~nm}$ and $1550 \mathrm{~nm}$ windows GaInAsN/ GaAs QW based semiconductor active layers is an alternative choice which can be easily grown on GaAs and offer larger conduction band offset compared to the above mentioned GaInAsP/ InP system [3]. Stronger electron confinement and improved high temperature performance has been obtained using this system [3].

Improvement in characteristics have been obtained by using AlGaInAs /InP active region which produce a larger 
conduction band offset than InGaAsP/InP active region [4]. $\mathrm{AlGaInAs} / \mathrm{InP} \mathrm{QWs}$, have better temperature performance than GaInAsP QWs [1].

A comparison of gain calculations among GaInAsN/ GaAs, AlGaInAs/ AlGaInAs and GaInAsP/ GaInAsP QW systems have been performed in [5] for long wavelength performance. After comparing the gain performance of InGaAsP, InGaAsN and AlGaInAs QWs of equal QW width, Yong et. al. [5] found that InGaAsN and AlGaInAs have extremely promising differential gain performance at $300 \mathrm{~K}$ and $375 \mathrm{~K}$, while maintaining low-transparency carrier density. According to Yong et. al. InGaAsN and AlGaInAs have good potential as active layer material systems for low- threshold and high speed modulation bandwidth devices required for next generation low cost communication links [5].

It has been found that the defect-related nonradiative recombination is significantly high in $\mathrm{InGaAsN}$ material due to the difficulty in the growth of high quality nitride compounds [6].

In this work, AlGaInAs has been chosen as the active material to get better performance. A new structure of VCSEL has been presented in this work which utilizes the benefits of intracavity structure and which has good mechanical strength and at the same time is capable of good heat dissipation because of heat sinking using extended metallization. In this work, starting from the substrate, bottom DBR mirror system up to the last layer of the active region, the VCSEL can be grown epitaxially as the layers are closely lattice matched except the introduction of small compressive strain in the QWs. As a whole, the fabrication of this VCSEL is kept simple including the fabrication of the 5 pairs of dielectric top DBR mirror system.

\section{Energy Sub-Bands in the Quantum Wells and Material Gain of the Active Region}

\subsection{Computation of Energy Sub-Bands in the Quantum Wells}

In this work, strain has been used in the QW due to which the shift in conduction band bottom edge and the shift in the sub-bands (heavy hole and light hole) of valence band are to be computed using strain. If $a$ and $a_{0}$ are lattice constants of well and barrier material respectively, then strain in in-plane direction can be written as [7]

$$
\varepsilon=\varepsilon_{x x}=\varepsilon_{y y}=\frac{a_{0}-a}{a}
$$

If $a_{0}>a$, then it is called tensile strain and if $a_{0}<a$, then it is called compressive strain.

The analytical expression for strain in the perpendicular direction is [6]

$$
\varepsilon_{z z}=-2 \frac{C_{12}}{C_{11}} \varepsilon
$$

where, $\mathrm{C}_{11}$ and $\mathrm{C}_{12}$ are shear elastic coefficients.

Conduction band-edge shift $\left(\delta E_{C}\right)$ is calculated using the analytical expression [7]

$$
\delta E_{C}=2 a_{c}\left(1-\frac{C_{12}}{C_{11}}\right) \mathcal{\varepsilon}
$$

The shift in the edges of valence band sub-bands ( $\delta E_{H H}$ and $\left.\delta E_{L H}\right)$ are expressed as [7],

$$
\delta E_{H H}=-P_{\varepsilon}-Q_{\varepsilon} \text { and } \delta E_{L H}=-P_{\varepsilon}+Q_{\varepsilon}
$$
where,

$$
P_{\varepsilon}=-2 a_{v}\left(1-\frac{C_{12}}{C_{11}}\right) \mathcal{\varepsilon} \text { and } Q_{\varepsilon}=-b\left(1+\frac{C_{12}}{C_{11}}\right) \varepsilon
$$

$a_{c}=$ conduction band deformation potential, $a_{v}=$ valence band deformation potential and $b=$ shear deformation potential

Effective masses of heavy-hole and light-hole band are also changed due to strain. Effective masses of heavy hole and light hole bands at growth direction under strain can be expressed as [8],

$$
m_{h h}=\frac{m_{h h 0}}{\gamma_{1}-2 \gamma_{2}} \text { and } m_{l h}=\frac{m_{l h 0}}{\gamma_{1}+2 \gamma_{2}}
$$

Where, $m_{h h 0}$ and $m_{l h 0}$ are effective masses of heavy hole and light hole band at unstrained condition and $\gamma_{1}, \gamma_{2}$ are Luttinger parameters.

For calculating the conduction sub-bands and valence sub-bands of the quantum well region of a VCSEL, the well known time independent Schrodinger's equation [9] has been used.

$$
-\frac{\hbar^{2}}{2 m_{e f f}} \nabla^{2} \Psi+V \Psi=E \Psi
$$

Where, $\Psi$ is the particle wave function, $m_{\text {eff }}$ is the effective mass of the carrier, $V$ is band potential and $E$ is the energy levels of conduction or valence band.

The band energy levels are calculated from the eigen values of the above equation.

\subsection{Computation of Material Gain of the Active Region}

The optical gain for a material is achievable over a narrow band of photon's energy. For the calculation of gain spectra of the active region of a VCSEL, the following well known gain expression [10] has been used.

$$
g(E)=g_{0}(E)\left|M_{b}\right|^{2}
$$




$$
\sum_{j, n} \int_{E_{g, j n}}^{E_{g, b}} \rho_{r, j n}\left(E^{\prime}\right) A_{i j} C_{i j}\left(f_{c}-f_{v}\right)_{E^{\prime}, j n} L\left(E-E^{\prime}\right) d E^{\prime}
$$

Here, $g_{0}$ is gain prefactor, $M_{b}$ is the momentum transition matrix element, $\rho_{r, j n}$ is the volume density states, $f_{c}$ and $f_{v}$ are the electron quasi-Fermi level functions in the conduction and valence bands respectively. $L(E)$ is the normalize Lorentzian lineshape function give in [9], $A_{i j}$ is angular anisotropy factor and $C_{i j}$ is the spatial overlap factor between the states $i$ and $j$.

In the above mentioned calculation, the actual transition energy between $\mathrm{CB}$ subband and VB subband is required which is computed by the known equation.

$$
E_{i j}=E_{g}+E_{c i}+E_{h h j}
$$

\section{Designing the Laser Structure}

\subsection{Designing the Active Region and the Cavity of the VCSEL}

The energy gap, $E_{g}$ of the quantum well material corresponding to the $1310 \mathrm{~nm}$ solid state laser is calculated to be $0.948 \mathrm{eV}$. To achieve the above mentioned wavelength of $1310 \mathrm{~nm}$ and the corresponding $E_{g}=0.948$ $\mathrm{eV}$, the GaAs based quaternary compound semiconductor material $\mathrm{Al}_{0.155} \mathrm{Ga}_{0.105} \quad \mathrm{In}_{0.74} \mathrm{As}$ has been chosen as the quantum-well material. The above mentioned composition chosen for the well material $\mathrm{Al}_{\mathrm{x}} \mathrm{Ga}_{\mathrm{y}} \mathrm{In}_{1-\mathrm{x}-\mathrm{y}}$ As to produce the desired bandgap gives rise to a $1.55 \%$ compressively strain (with InP). For calculating its bandgap the following equation given in Selmic et al. [11] is used

$$
\begin{aligned}
& E_{g}=0.572+1.571 x \\
& 1-x-y=0.74
\end{aligned}
$$

The calculated value of $E_{g}$ of the quantum well quaternary material $\mathrm{Al}_{0.155} \mathrm{Ga}_{0.105} \mathrm{In}_{0.74} \mathrm{As}$ is computed as $0.81 \mathrm{eV}$. Along with the conduction and valence band subbands the value of energy gap is $0.948 \mathrm{eV}$ which almost corresponds to $1310 \mathrm{~nm}$.

To achieve an acceptable band offset the barrier material has also been chosen to be a quaternary compound $\mathrm{Al}_{\mathrm{x}} \mathrm{Ga}_{\mathrm{y}} \mathrm{In}_{1-\mathrm{x}-\mathrm{y}} \mathrm{As}$ lattice matched to InP. The composition of the semiconductor $\mathrm{Al}_{0.267} \mathrm{Ga}_{0.203} \mathrm{In}_{0.53} \mathrm{As}$ as barrier material is calculated using the equation given in Selmic et al. [11].

$$
\begin{aligned}
& E_{g}=0.75+1.548 x, \\
& 1-x-y=0.53
\end{aligned}
$$

This barrier material produces an energy band gap of $1.16 \mathrm{eV}$. The material used in the $\mathrm{SCH}$ layer is same as the barrier layers.

The p-cladding material is chosen as $\mathrm{Al}_{0.48} \mathrm{In}_{0.52} \mathrm{As}$ ( $\mathrm{Si}$ doped) with an energy bandgap $E g=1.45$ and the $\mathrm{n}$-cladding material is chosen as $\mathrm{Al}_{0.48} \mathrm{In}_{0.52} \mathrm{As}$ ( $\mathrm{C}$ doped)
$\left(E_{g}=1.45\right)$ both are lattice matched. The energy gap for this composition of the materials has been computed using the equation [12]

$$
E_{g}=0.359+1.931 x+0.72 x^{2}
$$

The energy band profile of the complete VCSEL is presented in Fig. 1.

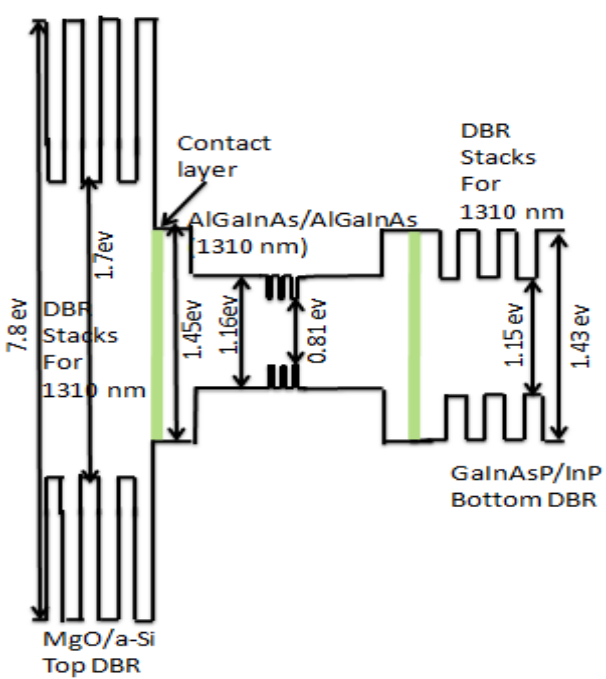

Figure 1. Energy band diagram showing three quantum wells, two barriers, two SCH regions, two cladding regions of the designed $1310 \mathrm{~nm}$ VCSEL using $A l_{0.155} \mathrm{Ga}_{0.105} \mathrm{In}_{0.74} \mathrm{As} / \mathrm{Al}_{0.267} \mathrm{Ga}_{0.203} \mathrm{In}_{0.53} \mathrm{As} \mathrm{MQW} /$ barrier. The forbidden energy gap of $0.81 \mathrm{eV}$ for the quantum well together with the subbands corresponds to the 1310nm lasing wavelength.

The conduction band offset $\Delta E_{c}$ for the chosen material has been computed as $0.32 \mathrm{ev}$, the valence band offset for heavy hole band $\Delta E_{h h}=0.0485$ and the valence band offset for light hole band $\Delta E_{l h}=0.12$ for the chosen material which are quite good.

The computed lattice constant of the quantum well material is 5.957 which has been computed using the equation $[11][13]$

$$
\begin{aligned}
& a_{A l_{x} G a_{y} I n_{1-x-y} A s}=(1-x-y) a_{I n A s}+x a_{A l A s} \\
& +y a_{G a A s}
\end{aligned}
$$

For the quantum well material the refractive index value is 3.42. The computed lattice constant of the barrier layer material is 5.869 and the refractive index value is 3.32 . The $\mathrm{SCH}$ material is the same as the barrier. The computed lattice constant of the p-cladding layer material is 5.869 and the refractive index value is 3.23 . The values are same for the n-cladding material. The composition of the contact layer material is the same as the cladding layers.

InP has been used as the substrate material whose lattice constant is 5.869 and the refractive index value is 3.18 . The obtained values of the refractive indices are shown in Fig. 2. 


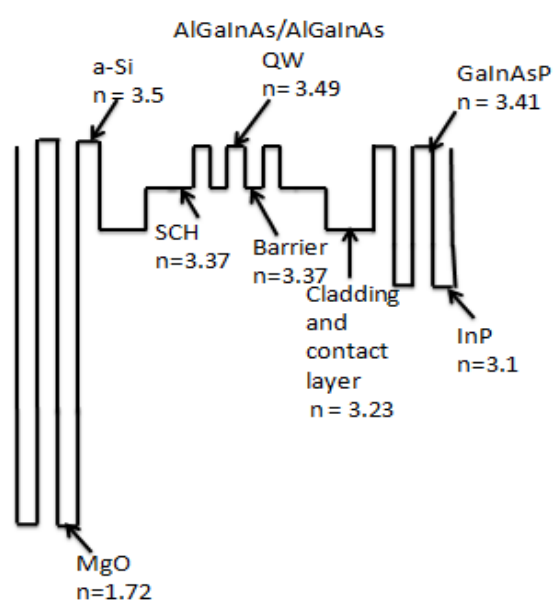

Figure 2. Refractive index values of the quantum wells, barriers, $S C H$, cladding layers and DBR layers of the top emitting 1310nm MQW VCSEL.

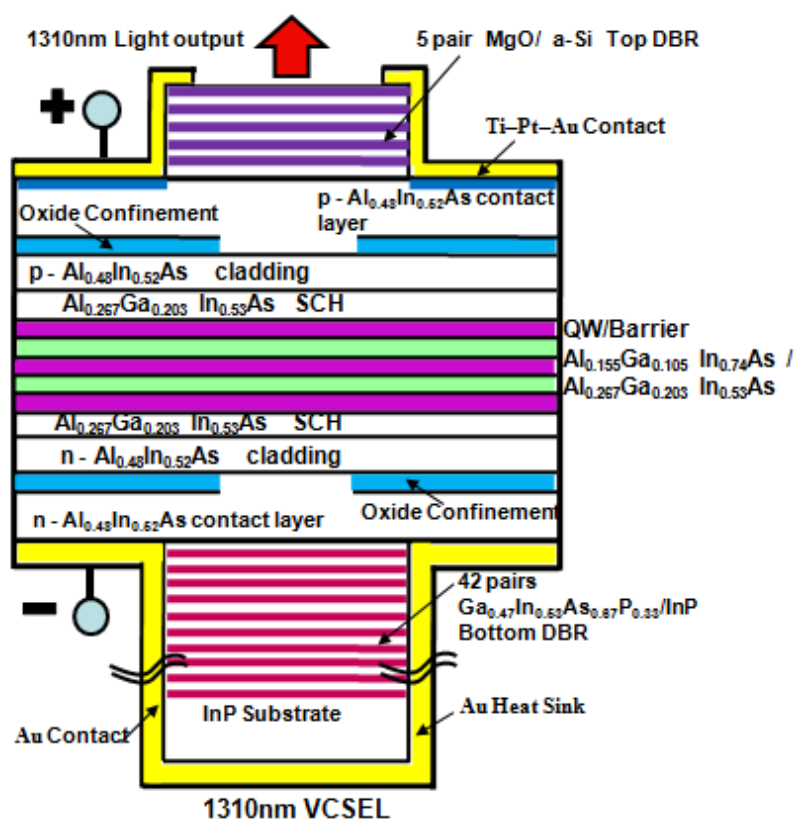

Figure 3. The designed 1310nm $A l_{0.155} \mathrm{Ga}_{0.105} \operatorname{In}_{0.74} \mathrm{As} / \mathrm{Al}_{0.3 \mathrm{G}} \mathrm{Ga}_{0.17} \mathrm{In} \mathrm{n}_{0.53} \mathrm{As}$ VCSEL using Intra Cavity Structure showing the materials used in the substrate, the layers of the active region, the DBR layers and the contact points.

The number of quantum wells used in this design is 3 . The barrier, the $\mathrm{SCH}$ and cladding are all fully lattice matched with the InP substrate material. The lattice constant of the quantum well material is nearly matched with these materials. As a result the entire VCSEL can be fabricated using epitaxial growth. The layers of the complete VCSEL are shown in Fig. 3.

\subsection{Computation of Material Gain}

While fixing the composition of the QW and barrier materials, computation of the energy subbands and the material gain have been done to ensure that the peak gain occurs at the desired wavelength of $1310 \mathrm{~nm}$. The finally obtained plot of the material gain vs. wavelength is presented in Fig. 4

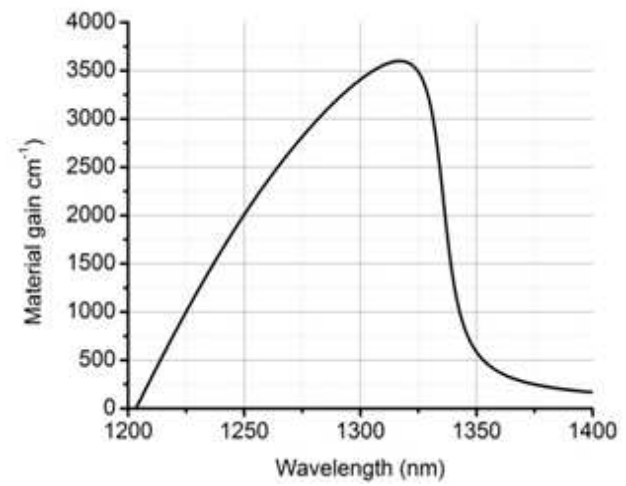

Figure 4. Plot of material gain versus wavelength for the designed VCSEL using $A l_{0.155} G a_{0.105} I_{0.74} A s / A l_{0.267} G a_{0.203} I_{0.53} A s$ QW/ barrier. The peak value of the material gain can be seen to occur at a value of wavelength of $1310 \mathrm{~nm}$.

\subsection{Fixing Cavity Length, Cavity Radius and Cavity Volume}

For this VCSEL, the designed cavity length is taken as $1.5 \lambda$ i.e., $1965 \mathrm{~nm}$. For this design, the physical thickness of each of the three identical quantum wells has been chosen as $7 \mathrm{~nm}$ which corresponds to an electronic thickness of $24.43 \mathrm{~nm}$. The thickness of each of the two identical barrier regions has been chosen as $10 \mathrm{~nm}$ which corresponds to an electronic thickness of $33.7 \mathrm{~nm}$ and the thickness each of the two $\mathrm{SCH}$ regions as $115 \mathrm{~nm}$ which corresponds to an electronic thickness of $387.55 \mathrm{~nm}$. The thickness of each of the two identical cladding (along with the contact layers) layers has been chosen as $162 \mathrm{~nm}$ which corresponds to an electronic thickness of $523.26 \mathrm{~nm}$. The structure is fully symmetric as a result the QWs are positioned at the antinode of the Electric field standing wave pattern. The Cavity length is then obtained by adding the thicknesses of the layers as $=1962 \mathrm{~nm}\left(L_{\text {cavity }}\right)$ which is very close to 1.5 wavelength i.e., $1965 \mathrm{~nm}$. The thickness of each of the $\mathrm{Al}_{\mathrm{x}} \mathrm{In}_{\mathrm{y}} \mathrm{O}_{\mathrm{z}}$ (oxide) confinement layers has been chosen as $15 \mathrm{~nm}$. The substrate thickness is chosen as $400 \mu \mathrm{m}$.

The QWs are placed so that the antinode of the field standing wave pattern is positioned on the middle QW as shown in the Fig. 5 .

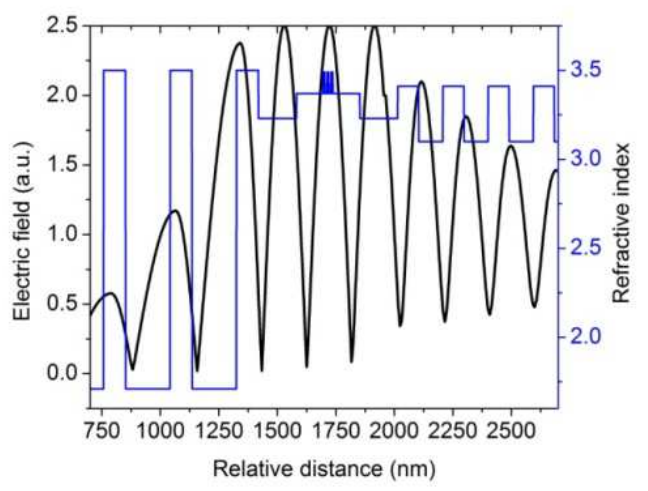

Figure 5. The field standing wave pattern along the length of the VCSEL excluding some pairs of top as well as bottom DBR mirror system. The refractive index vs. length plot is superimposed. 
The value of $L_{\text {active }}$ is the length of the three quantum wells which is $73.29 \mathrm{~nm}$ (optical length).

The active region radius is taken as $5 \times 10^{-4} \mathrm{~cm}$. The active volume then becomes $V_{\mathrm{a}}=1.6493 \times 10^{-12} \mathrm{~cm}^{3}$.

The value of transparency carrier density is $1.1 \times 10^{18} / \mathrm{cm}^{3}$ is obtained from the plot of material gain versus carrier density shown in the Fig. 6.

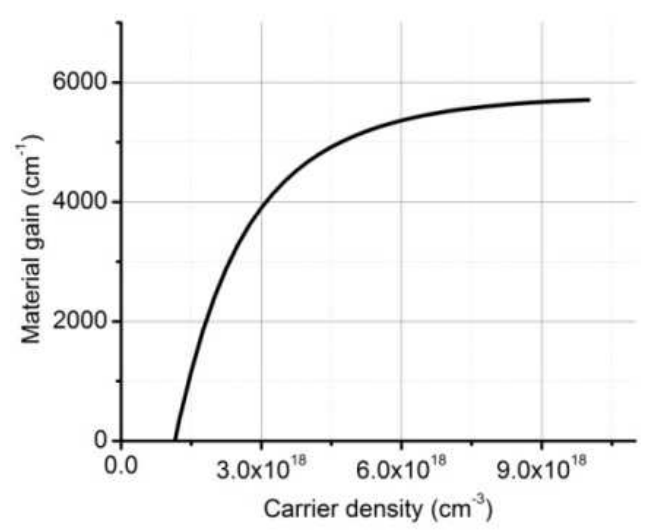

Figure 6. Plot of material gain versus carrier density for active region of the designed VCSEL

\subsection{Computation of Optical Confinement Factor Threshold Carrier Density Nth of the VCSEL}

The optical confinement factor with an enhancement factor of 2, assuming 90\% confinement in the transverse direction is represented as [14][15],

$$
\Gamma=2 \times \frac{L_{\text {active }}}{L_{\text {eff }}} \times 0.9
$$

The value of computed optical confinement factor is 0.0254 .

For a transparency carrier density $\left(N_{t r}\right)$ of $1.1 \times 10^{18} / \mathrm{cm}^{3}$, the carrier density at threshold $\left(N_{t h}\right)$ is computed to be $2.293 \times 10^{18} \mathrm{~cm}^{-3}$ using the following expression [14],

$$
N_{t h}=N_{t r} \times \exp \left(\frac{\alpha_{i}+\alpha_{m}}{\Gamma g_{0}}\right)
$$

Where, $\alpha_{i}=$ internal loss, $\alpha_{m}=$ mirror loss and $g_{0}=$ gain coefficient.

\subsection{Computation of Photon Life Time Tp of the VCSEL}

Using the following equation [15], the photon lifetime $\tau_{p}$ is computed to be $1.6439 \mathrm{ps}$

$$
\tau_{p}=\frac{1}{v_{g}\left(\alpha_{i}+\alpha_{m}\right)}
$$

\subsection{Computation of Threshold Current $I_{\text {th }}$ of the VCSEL}

The threshold current of the designed VCSEL is computed using the expression [15],

$$
I_{t h}=\frac{q V_{a} N_{t h}}{\eta_{i} \tau_{c}}
$$

For a current injection efficiency $\left(\eta_{i}\right)$ of 0.8 , a carrier lifetime $\tau_{c}=4 \times 10^{-9} \mathrm{sec}$, a threshold carrier density of $2.293 \times 10^{18} \mathrm{~cm}^{-3}, V_{\mathrm{a}}=1.6493 \times 10^{-12} \mathrm{~cm}^{3}$, the threshold current $\left(I_{t h}\right)$ is found to be $0.4 \mathrm{~mA}$ at room temperature.

\subsection{Calculation of Differential Gain}

Differential gain is obtained by differentiating the material gain $(g)$ with respect to carrier density. The value of differential gain at threshold carrier density of $2.293 \times 10^{18} \mathrm{~cm}^{-3}$ is obtained from the plot of differential gain vs. carrier density of Fig. 7 as $20 \times 10^{-16} \mathrm{~cm}^{2}$.

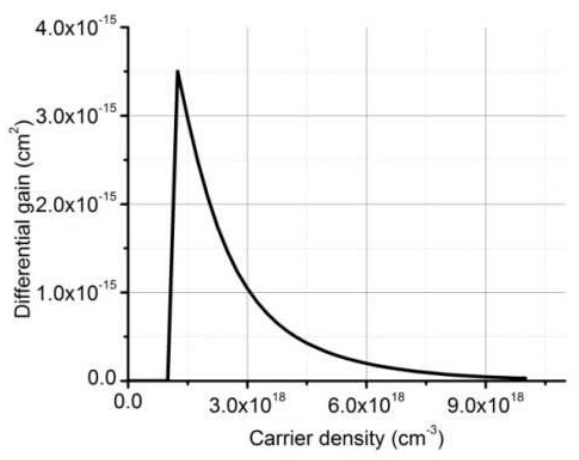

Figure 7. Plot of differential gain vs. carrier density for the compressively strained quantum well material system used in the active region of the designed VCSEL.

\subsection{The Bottom DBR Mirror System of the VCSEL}

The design has been based on building the laser on a $500 \mu \mathrm{m}$ thick InP substrate. The bottom DBR mirror system is chosen to be 42 pairs of lattice matched $\mathrm{Ga}_{0.47} \mathrm{In}_{0.53} \mathrm{As}_{0.67} \mathrm{P}_{0.33} / \mathrm{InP}$ and matched to InP which is grown on the InP substrate. This system gives a reflectivity of $99.9 \%$. The refractive index of $\mathrm{Ga}_{0.47} \mathrm{In}_{0.53} \mathrm{As}_{0.67} \mathrm{P}_{0.33}$ is 3.41 and that of InP is 3.1. The thickness of each layer of the $\mathrm{Ga}_{0.47} \mathrm{In}_{0.53} \mathrm{As}_{0.67} \mathrm{P}_{0.33}$ Bragg reflector material has been designed as $\lambda / 4 n_{h}=1310 /\{4 \times 3.41\} \mathrm{nm}=96.04 \mathrm{~nm}$ and the thickness of each layer of the InP Bragg reflector material has been designed as $\lambda / 4 n_{l}=1310 /\{4 \times 3.1\} \mathrm{nm}=105.6 \mathrm{~nm}$. A reflectivity of $99.9 \%$ is obtained from this top DBR system.

\subsection{The Top DBR Mirror System of the VCSEL}

The top DBR mirror system has been formed by fabricating 5 pairs of $\mathrm{MgO} / \mathrm{a}-\mathrm{Si}$ which has a high refractive index contrast of 1.78. A reflectivity of $99.4 \%$ is obtained from the top DBR system. The refractive index of a-Si is 3.5 and that of $\mathrm{MgO}$ is 1.72 . The thickness of each layer of the a-Si Bragg reflector material has been designed as $\lambda / 4 n_{h}$ $=1310 /\{4 \times 3.5\} \mathrm{nm}=93.57 \mathrm{~nm}$ and the thickness of each layer of the $\mathrm{MgO}$ Bragg reflector material has been designed as $\lambda / 4 n_{l}=1310 /\{4 \times 1.72\} \mathrm{nm}=190.41 \mathrm{~nm}$. A reflectivity of $99.4 \%$ is obtained from this top DBR system. 
Following the equations given in Sale [16] the reflectivity values have been computed for the top and the bottom DBR regions. The plots are presented in Fig. 8 and 9.

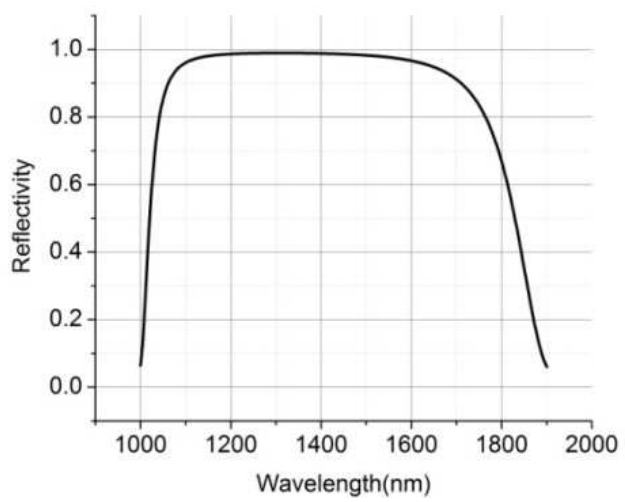

Figure 8. Plot of Reflectivity vs. wavelength for the top DBR region for obtaining $99.4 \%$ reflectivity using 5 pairs of $\mathrm{MgO} / \mathrm{a}$-Si dielectric materials of the designed 1310nm VCSEL.

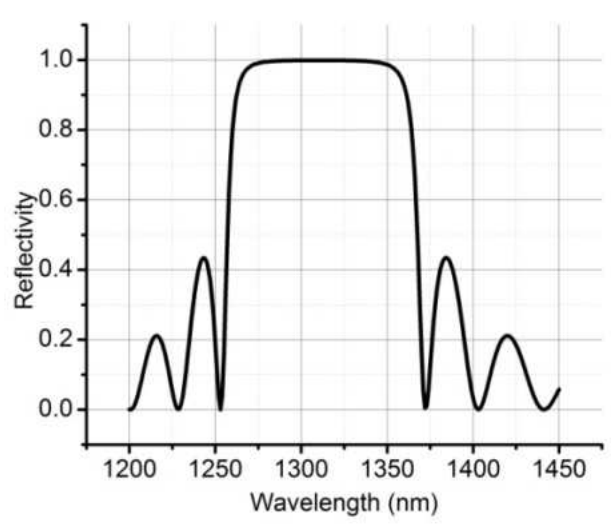

Figure 9. Plot of Reflectivity vs. wavelength for the bottom DBR region for obtaining $99.9 \%$ reflectivity using 42 pairs of $\mathrm{Ga}_{0.47} I n_{0.53} A s_{0.67} P_{0.33} / \operatorname{InP}$ material matched to InP of the designed $1310 \mathrm{~nm}$ VCSEL

\section{Computation of the Performance Characteristics}

Using the parameter values of the designed VCSEL, the solutions to the well known coupled rate equations (for the carrier density and the photon density) has been obtained for a time window of 0 - 3ns approximately using MATLAB for a chosen (practical) value of injection current $(10 \mathrm{~mA})$. The value of the injection current is to be taken above the threshold current.

\subsection{The Characteristics of the Designed VCSEL}

Using the output of this computation work a plot of carrier density versus time is obtained for the designed 1310nm VCSEL which is presented in Fig. 10. Using the output of the same computation work mentioned above, a plot of photon density versus time is obtained for the designed 1310nm VCSEL which is presented in Fig. 11.

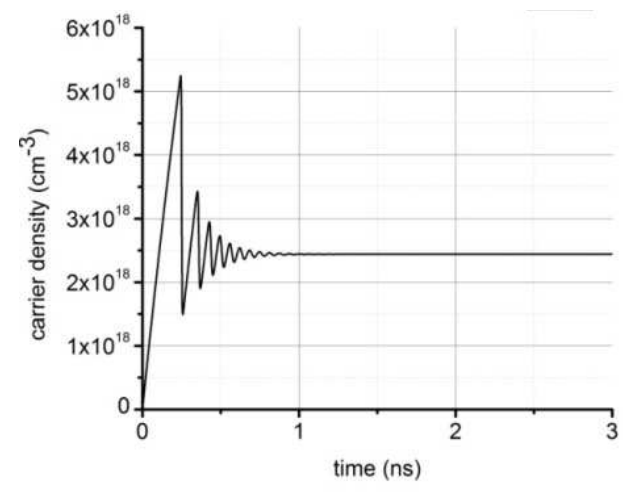

Figure 10. Plot of carrier density versus time for the designed $1310 \mathrm{~nm}$ VCSEL for an injection current value of $10 \mathrm{~mA}$

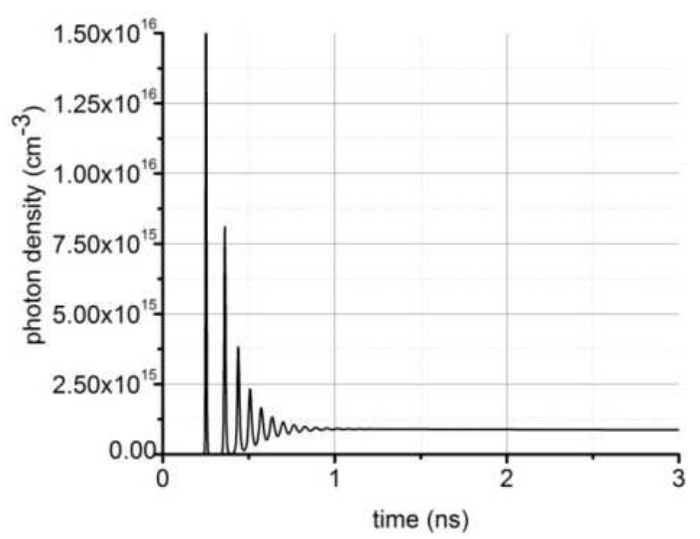

Figure 11. Plot of photon density versus time for the designed $1310 \mathrm{~nm}$ VCSEL for an injection current value of $10 \mathrm{~mA}$ and for the parameter values presented in the design section.

The output power of the 1310nm VCSEL is calculated using the well known equation [15]

$$
P_{\text {out }}=\frac{v_{g} \alpha_{m} h v V_{a} S}{\Gamma}
$$

where, $v_{g}=$ group velocity, $\alpha_{m}=$ mirror loss, $V_{a}=$ active region volume, $S=$ photon density and $\Gamma=$ confinement factor. Using this equation and the parameter values presented above plots of the output power versus injection current of the designed $1310 \mathrm{~nm}$ VCSEL is obtained through computation for a number of values of temperature. These plots of output power versus injection current are presented in Fig. 12. The VCSEL is designed for single transverse mode output $\left(\mathrm{TEM}_{00}\right)$ in order to achieve good beam quality. It shows output power of about $2.5 \mathrm{~mW}$ for an injection current of $16 \mathrm{~mA}$ at room temperature. J. Cheng et. al. [17] demonstrated a single mode VCSEL which shows peak output power a bit lower than our designed VCSEL (1.68 $\mathrm{mw}$ at room temperature). Moreover, the threshold current of our designed VCSEL (0.4 mA) is lower than the threshold current of VCSEL demonstrated in that paper [17]. The plot of output power versus wavelength is presented in Fig. 13. 


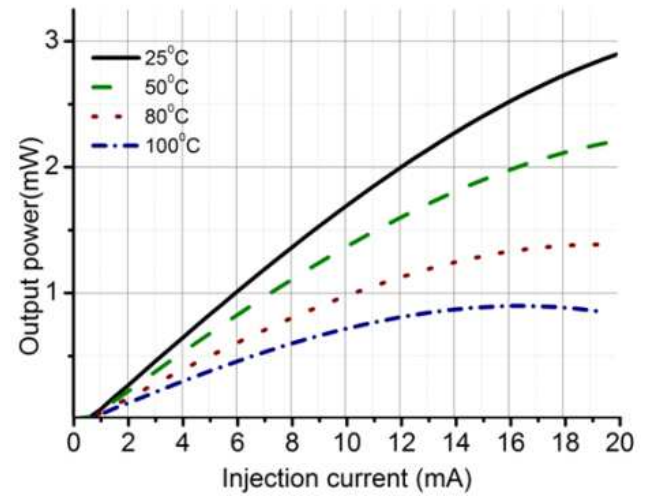

Figure 12. Plot of output power versus injection current for the designed $1310 \mathrm{~nm}$ VCSEL for different values of temperature. The threshold current is $0.4 \mathrm{~mA}$ at $25^{\circ} \mathrm{C}$ as can be seen from the plot.

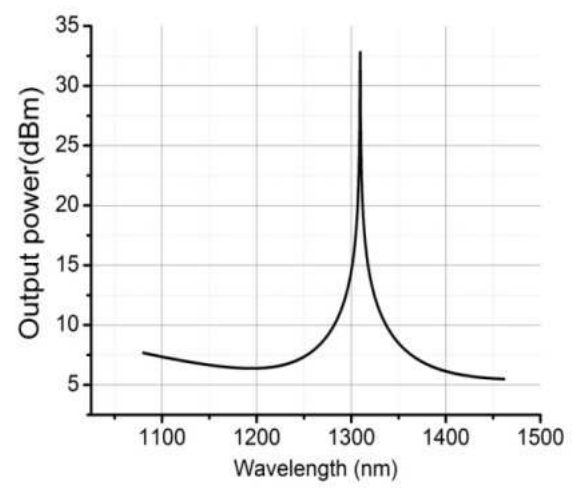

Figure 13. Plot of output power versus injection current for the designed $1310 \mathrm{~nm}$ VCSEL at an injection current of $10 \mathrm{~mA}$.

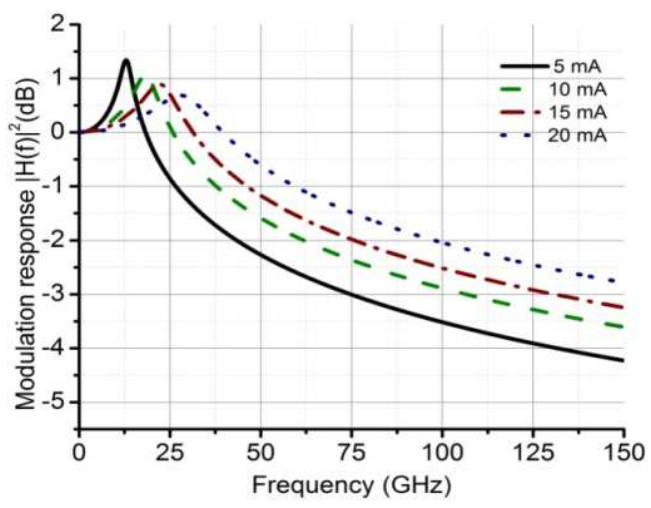

Figure 14. Plot of modulation transfer function versus wavelength for the designed $1310 \mathrm{~nm}$ VCSEL.

\subsection{High Speed Operation of the Designed VCSEL}

For the designed $1310 \mathrm{~nm}$ VCSEL a high value of differential gain of $20 \times 10^{-16} \mathrm{~cm}^{2}$ has been obtained because of the use of small amount of compressive strain of $1.55 \%$. Such a high value of differential gain indicates that the relaxation frequency is high which in turn indicates capability of high speed operation.

The modulation response of the designed laser has been obtained using the equation of the transfer function given in [15] for a number of values of injection current. The plots are presented in Fig. 14. From the plots it may be observed that the relaxation frequency for a low current of $5 \mathrm{~mA}$ is $12.5 \mathrm{GHz}$ and for a normal current of $10 \mathrm{~mA}$ this value is $20 \mathrm{GHz}$ which indicates capability of satisfactory high speed data communication. The $3 \mathrm{~dB}$ bandwidth at $10 \mathrm{~mA}$ is found to be $100 \mathrm{GHz}$.

\section{Conclusions}

A 1310nm VCSEL has been designed using quaternary compound of AlGaInAs in the QW and barrier. The QW is $1.55 \%$ compressively strained. The rest of the layers except the top DBR mirror system are fully lattice matched to the substrate InP. As a result, from the substrate up to the top DBR the VCSEL can be easily fabricated using epitaxial growth. This is achieved at the expense of a long bottom DBR mirror system. However, this length is not contributing in raising the VCSEL interior temperature as the injection current is not flowing through the DBR mirror systems.

The top dielectric DBR layers have reduced the height of the VCSEL significantly because only 5 pairs are required. These dielectric DBR layers can be grown using evaporation technique. With this combination of mirrors and with the chosen material the overall fabrication procedure remains simple which is an attraction of this VCSEL. This particular intra-cavity structure and the shape of the VCSEL along with the two oxide confinement layer will ensure the required current confinement and the design of metallization will help to dissipate the heat easily. The present structure can be used as a discrete laser. However, it can also be used in a two dimensional array with little modification.

The computed performance characteristic curves show good performance. The threshold current is on the low side which is desired. The power vs. injection current characteristics at higher temperature of the VCSEL is good. Good value of differential gain has been achieved because of the use of small compressive strain in the QW. This in turn has given rise to higher relaxation frequency. This will provide high speed performance of the VCSEL.

\section{References}

[1] N. Nishiyama, C. Caneau, B. Hall, G. Guryanov, M. H. Hu, X. S. Liu, M.-J. Li, R. Bhat, and C. E. Zah,"Long-Wavelength Vertical-Cavity Surface-Emitting Lasers on InP With Lattice Matched AlGaInAs-InP DBR Grown by MOCVD", IEEE Journal Of Selected Topics In Quantum Electronics, Vol. 11, No. 5, September/October 2005, pp. 990-998.

[2] Kenichi Iga,"Vertical-Cavity Surface-Emitting Laser: Its Conception and Evolution", Japanese Journal of Applied Physics, Vol. 47, No. 1, 2008, pp. 1-10.

[3] Z. Pan, L. H. Li, Y. W. Lin, B. Q. Sun, D. S. Jiang and W. K. Ge,"Conduction band offset and electron effective mass in GaInNAs/ GaAs quantum-well structures with low nitrogen concentration", Applied Physics Letters Volume 78, Number 15, 9 April 2001, pp. 2217-2219. 
[4] Joachim Piprek, Manish Mehta, and Vijay Jayaraman," Design and Optimization of High-Performance $1.3 \mu \mathrm{m}$ VCSELs", Physics and Simulation of Optoelectronic Devices XII, Proceedings of SPIE Vol. 5349, 2004, pp. 375-384.

[5] J. C. L. Yong, Judy M. Rorison, and Ian H. White,"1.3- m Quantum-Well InGaAsP, AlGaInAs, and InGaAsN Laser Material Gain: A Theoretical Study", IEEE Journal of Quantum Electronics, Vol. 38, No. 12, December 2002, pp. $1553-1564$

[6] S. R. Jin, S. J. Sweeney, S. Tomic, A. R. Adams, and H. Riechert, "Unusual increase of the Auger recombination current in $1.3 \mathrm{~mm}$ GaInNAs quantum well lasers under high pressure," Appl. Phys. Lett., vol. 82, April 2003 pp. 2335-2337.

[7] S. Lien Chuang, "Physics of Optoelectronic Devices," 1995, Wiley-Interscience, p. 442.

[8] J. Piprek, "Semiconductor optoelectronic devices: Introduction to Physics and Simulation," 2003, Academic Press, pp. 24-26

[9] P. Harrison, "Quantum Wells, Wires and Dots: Theoretical and Computational Physics of Semiconductor Nanostructures", John Wiley \& sons, West Sussex, 2009, pp. 17-70, 73-82.

[10] P. S. Zory, "Quantum Well Lasers" Academic Press, London, 1993, pp. 28-41, 132-154.

[11] S. R. Selmic, T. M. Chou, J. P. Sih, J. B. Kirk, A. Mantie, J.
K. Butler, D. Bour, and G. A. Evans, "Design and characterization of 1.3- $\mu \mathrm{m}$ AlGaInAs-InP Multiple quantum well lasers," IEEE Journal on Selected Topics in Quantum Electronics, Vol. 7, No. 2, March/April 2001, p. 342.

[12] S. Adachi, "Properties of Semiconductor Alloys Group-IV, III-V, II-VI Semiconductor", John Wiley \& Sons, New York, 2009, pp. 133-214, 238-253, 277-286, 307-332.

[13] I. Vurgaftman, J. R. Meyer and L. R. Ram-Mohan, "Band parameters for III-V compound semiconductors and their alloys", Journal of Applied Physics, Vol. 89, No. 11, 1 June 2001 Appl. Phys. Rev.: Vurgaftman, Meyer, and Ram-Mohan, pp. 5815-5875.

[14] Vlad Badilita," Study of Vertical Coupled-Cavity Laser Structures", Doctor of Science Thesis, Institut de photonique et d'électronique quantiques, Physics Department, École Polytechnique Fédérale De Lausanne, Université de Bucarest, Roumanie et de nationalité Roumaine, 2004, p. 50.

[15] L. A. Coldren and S. W. Corzine, "Diode Lasers and Photonic Integrated Ciruits", John Wiley \& Sons, N.Y., 1995 , pp. 30-59, 186-250.

[16] T. E. Sale, "Vertical Cavity Surface Emitting Lasers" John Wiley \& Sons Inc., 1995, pp. 33-35.

[17] J. Cheng, C. L. Shieh, X. D. Huang, G. L. Liu, M. V. R. Murty, C. C. Lin, and D. X. Xu., "Efficient long wavelength AlGaInAs vertical-cavity surface-emitting lasers for coarse WDM applications over multimode fibre." Electronics Letters, Vol. 40, No. 19, September 2004, pp 1184-1185. 